\title{
Macrolides and Torsadogenic Risk: Emerging Issues from the FDA Pharmacovigilance Database
}

Emanuel Raschi ${ }^{1}$, Elisabetta Poluzzi ${ }^{1}$, Ariola Koci ${ }^{1}$, Ugo Moretti ${ }^{2}$, Miriam Sturkenboom ${ }^{3}$ and Fabrizio De Ponti ${ }^{1 *}$

${ }^{1}$ Department of Medical and Surgical Sciences, Pharmacology Unit, Alma Mater Studiorum, University of Bologna, Italy

${ }^{2}$ Clinical Pharmacology Unit, University of Verona, Italy

3Erasmus Medical Center, Rotterdam, Netherlands

\begin{abstract}
Introduction: Concern exists on the pro-arrhythmic potential of macrolides, namely Torsade de Pointes (TdP). Recent evidence has challenged the common opinion of considering azithromycin a safer therapeutic option, causing emerging regulatory and clinical interest.

Materials and Methods: We analyzed cases of drug-induced TdP (2004-2011) submitted to the publicly available FDA Adverse Event Reporting System (FAERS). Four groups of mutually exclusive events were identified in decreasing order of drug-attributable risk: 1) TdP; 2) QT interval abnormalities; 3) ventricular arrhythmia (VA); 4) Sudden Cardiac Death (SCD). They were combined into case definition A (TdP/QT abnormalities) and case definition $B(V A / S C D)$. Both case-by-case analysis (information on concomitant drugs, especially QT-prolonging agents listed by Arizona CERT, and disproportionality approach (Reporting Odds Ratio, ROR, with $95 \% \mathrm{Cl}$ ) were carried out.

Results: Over the 8-year period, macrolides were associated with 183 and 419 cases of interest (case definition $A$ and $B$, respectively). Clarithromycin was the most frequently reported (84 and 162 cases), followed by azithromycin (63 and 140). Only $27 \%$ of cases of TdP/QT abnormalities with azithromycin occurred in patients $>65$ years of age (63, 47 and $44 \%$ for clari-, ery- and telithromycin, respectively). In cases of TdP/QT abnormalities, concomitant QT-prolonging drugs (Arizona CERT lists 1 or 2) were recorded with a proportion very different among macrolides (11 to $89 \%)$. The highest percentage of fatal outcome was recorded for azithromycin (17\%). Disproportionality was found for azithromycin, clarithromycin and telithromycin for both events of interest, whereas erithromycin showed disproportion only for TdP/QT abnormalities.

Conclusions: Despite inherent limitations of spontaneous reporting analyses, the remarkable proportion of fatal cases and the occurrence of TdP-related events in middle-aged patients strengthen the view that caution is needed before considering azithromycin as a safer therapeutic option among macrolides.
\end{abstract}

Keywords: Spontaneous reporting systems; Disproportionality; Azithromycin

\section{Introduction}

Macrolides are a class of concern for arrhythmia, especially Torsade de Pointes (TdP) and its surrogates: QT interval prolongation, ventricular arrhythmia (VA), such as ventricular tachycardia, fibrillation and subsequent Sudden Cardiac Death (SCD) [1]. It is generally believed that erythromycin (in particular, intravenously) carries the highest risk, while azithromycin is thought to have only a minimal effect on the cardiovascular system [2].

However, a recent cohort study by Ray et al. [3], found increased risk of cardiovascular death during a 5-day azithromycin treatment (as compared to amoxicillin and ciprofloxacin, with a risk similar to levofloxacin). Because no formal comparison was undertaken among macrolides, the question arises whether or not we are dealing with a class effect. Notably, the FDA is reviewing this safety aspect for potential regulatory measures with related clinical implications. In addition, several drug utilization studies underlined an increased use in the population over the past decade, both in US and Europe, a scenario suggesting inappropriate prescription [4-8].

Therefore, we provide the contribution of pharmacovigilance by critically exploring the FDA Adverse Event Reporting System (FAERS). Despite inherent limitations, FAERS offers public access to raw data, covers virtually the entire population (by including US reports and serious/unexpected events from Europe) and has been recently used to investigate rare events such as $\mathrm{TdP}$ that may otherwise be undetected in dedicated clinical trials $[9,10]$. In addition, interest is emerging in using FAERS to obtain reliable within-class comparisons $[11,12]$.

\section{Materials and Methods}

\section{Case definition}

Taking into account the multifaceted clinical presentation of TdP, a multidisciplinary panel of experts (i.e., cardiology, pharmacoepidemiology and pharmacovigilance reached consensus on definition of drug-induced TdP, by identifying 4 groups of events reflecting a decreasing order of drug-attributable risk: 1) TdP; 2) QT interval abnormalities (including QT prolongation and shortening); 3) ventricular arrhythmia (VA, including ventricular fibrillation/ tachycardia); 4) Sudden Cardiac Death (SCD). These groups were mutually exclusive (i.e. a single case report of arrhythmias was classified

*Corresponding author: Fabrizio De Ponti, Department of Medical and Surgical Sciences, Pharmacology Unit, University of Bologna, Via Irnerio, 48, I-40126 Bologna BO, Italy, Tel: +39-051-2091805; Fax: +39-051-248862; E-mail: fabrizio.deponti@unibo.it

Received January 26, 2013; Accepted February 11, 2013; Published February 13, 2013

Citation: Raschi E, Poluzzi E, Koci A, Moretti U, Sturkenboom M, et al. (2013) Macrolides and Torsadogenic Risk: Emerging Issues from the FDA Pharmacovigilance Database. J Pharmacovigilance 1: 104. doi:10.4172/ 2329-6887.1000104

Copyright: (C) 2013 Raschi E, et al. This is an open-access article distributed under the terms of the Creative Commons Attribution License, which permits unrestricted use, distribution, and reproduction in any medium, provided the original author and source are credited. 
Citation: Raschi E, Poluzzi E, Koci A, Moretti U, Sturkenboom M, et al. (2013) Macrolides and Torsadogenic Risk: Emerging Issues from the FDA Pharmacovigilance Database. J Pharmacovigilance 1: 104. doi:10.4172/2329-6887.1000104

Page 2 of 4

only in one group with the following priority: $1>2>3>4$ ). In this study, these four groups were combined as follows: TdT with QT abnormalities (case definition A), VA with SCD (case definition B).

Before performing the analysis, several technical issues were handled with. This is the case for the drug mapping, duplicate removal and management of missing data [13]. Data of interest for the 20042011 period were collected from "DEMO" (demographic information), "DRUG" "REACTION" (adverse events coded according to the Medical Dictionary for Regulatory Activities terminology, MedDRA version 13.0) and "OUTCOME" files [14].

\section{Data analysis}

In the light of the peculiar clinical setting in which TdP usually occurs (i.e., multi-factorial causative roles with different risk factors), a case listing was first generated in order to describe demographic information (e.g., age and sex) and concomitant drugs, which may act as potential confounders of the drug-event association by reducing the so-called "repolarization reserve" [15]. Specifically, each event of interest was assessed for co-administration of drugs with cardiovascular indications (i.e., Class III antiarrhythmic drugs, proxy of already diagnosed arrhythmia; digitalis, diuretics, beta blockers, calcium channel blockers and ACE inhibitors/ARBs, proxies of heart disease) and agents with TdP liability listed by the AZCERT website (as of November $9^{\text {th }}, 2012$ ). In addition, the seriousness of the event was retrieved by checking information on the outcome (i.e., whether or not the event was fatal).

Moreover, disproportionality analysis was performed by calculating the Reporting Odds Ratio (ROR), with corresponding 95\% Confidential Interval (CI). Statistical significance was formally defined when the lower limit of the $95 \%$ Confidence Interval (95\%CI) was $>1$, with at least 3 cases. We gained insight into the temporal appearance of disproportionality for azithromycin and TdP/QT abnormalities by providing a cumulative time-series analysis of the ROR [16].

Only reports where macrolides were recorded as suspect (e.g., "primary" or "secondary" suspect) or interacting drug were considered. In addition, reports with missing information on age and gender were excluded. The statistical package SPSS (version 19.0) was used.

\section{Results}

Over the 8-year period, 2,679,762 spontaneous reports were retrieved after removal of duplicates and multiple records. Overall, a total of 7,844 (6,056 with at least one active substance) and 72,366 (55,854 with at least one active substance) cases were retrieved (case definition $\mathrm{A}$ and $\mathrm{B}$, respectively). Macrolides, lincosamides and streptogramins (J01F) were reported in 197 and 480 events of interest ( $A$ and $B$, respectively).

Table 1 shows the complete case listing (i.e., demographic data, information on concomitant drugs and is proportionality analysis). Macrolides were associated with 183 and 419 cases of interest (TdP/QT abnormalities and VA/SCD, respectively). Clarithromycin was the most frequently reported drug ( 84 and 162 cases), followed by azithromycin (63 and 140).

Most of the cases occurred in females. In addition, only $27 \%$ of cases of TdP/QT abnormalities with azithromycin occurred in patients of $>65$ years of age, whereas this percentage was much higher for clari-, ery- and telithromycin: 63,47 and 44 , respectively.

Remarkably, QT-prolonging drugs of the Arizona CERT lists 1 or 2 were concomitantly recorded in $59 \%$ of cases of TdP/QT abnormalities associated with azithromycin $(45,89$ and $11 \%$, with clari-, ery- and telithromycin, respectively). Concomitant drugs with cardiovascular indication were reported in $22 \%$ of cases with azithromycin $(21,10$ and $5 \%$, with the other macrolides). Fatal outcome was found in $17 \%$ of azithromycin cases and in 4,11 and $11 \%$ with clari-, ery- and telithromycin, respectively.

A disproportionality signal was found for azithromycin, clarithromycin and telithromycin for both events of interest. Erythromycin showed disproportion only for TdP/QT abnormalities; the 31 cases of VA/SCD did not provided statistically significant ROR.

The time-trend analysis showed a steady number of TdP/QT abnormality reporting in the last 4 years; disproportionality was already significant in 2007-Q2 (ROR=2.15; 95\% CI=1.07-4.32; Figure 1) and ROR values increased up to $5.02(95 \% \mathrm{CI}=3.47-7.26)$ in $2010-\mathrm{Q} 1$.

\section{Discussion}

All the widely used macrolides, including azithromycin, are

Table 1: Data mining of the FAERS database (2004-2011 period), Only macrolides with at least one case of interest are shown.

\begin{tabular}{|c|c|c|c|c|c|c|c|}
\hline Macrolide (AZCERT List) & N. cases $(\% \mathrm{~F})$ & $\begin{array}{c}\text { Age }(0-17 ; 18-65 ; \\
>65)\end{array}$ & By MD & $\begin{array}{l}\text { N. cases with } \\
\text { c.v. drugs\# }\end{array}$ & $\begin{array}{l}\text { N. cases with } \\
\text { AZCERT drugs } \\
\text { (List } 1 ; 2 ; 3) \text { \# }\end{array}$ & N. Fatal cases & ROR $(95 \% \mathrm{Cl})$ \\
\hline \multicolumn{8}{|l|}{ TdP+QT Abnormalities } \\
\hline Azithromycin (I) & $63(67)$ & $3 ; 43 ; 17$ & 30 & 14 & $23 ; 11 ; 3$ & 11 & $5.69(4.43-7.31)^{*}$ \\
\hline Clarithromycin (I) & $84(55)$ & $3 ; 28 ; 53$ & 33 & 18 & $37 ; 18 ; 1$ & 3 & $6.23(5.01-7.74)^{\star}$ \\
\hline Erythromycin (I) & $19(68)$ & $2 ; 8 ; 9$ & 6 & 2 & $8 ; 3 ; 6$ & 2 & $5.28(3.35-8.32)^{\star}$ \\
\hline Telithromycin (II) & $18(78)$ & $0 ; 10 ; 8$ & 7 & 1 & $0 ; 1 ; 1$ & 2 & $3.35(2.11-5.34)^{\star}$ \\
\hline Roxithromycin (II) & $1(0)$ & $0 ; 1 ; 0$ & 1 & 0 & $0 ; 1 ; 0$ & 0 & n.a. \\
\hline Spiramycin (n.r.) & $1(100)$ & $0 ; 0 ; 1$ & 0 & 1 & $0.0 ; 0$ & 0 & n.a. \\
\hline \multicolumn{8}{|l|}{ VA+SCD } \\
\hline Azithromycin (I) & $140(54)$ & $16 ; 94 ; 30$ & 66 & 6 & $13 ; 24 ; 14$ & 54 & $1.35(1.14-1.60)^{*}$ \\
\hline Clarithromycin (I) & $162(54)$ & $6 ; 86 ; 70$ & 48 & 11 & $15 ; 35 ; 12$ & 63 & $1.28(1.09-1.49)^{*}$ \\
\hline Erythromycin (I) & $31(48)$ & $6 ; 14 ; 11$ & 10 & 3 & $3 ; 9 ; 0$ & 15 & $0.91(0.64-1.31)$ \\
\hline Telithromycin (II) & $89(61)$ & $0 ; 60 ; 29$ & 52 & 3 & $8 ; 7 ; 4$ & 27 & $1.83(1.48-2.27)^{\star}$ \\
\hline Roxithromycin (II) & $7(43)$ & $0 ; 2 ; 5$ & 3 & 0 & $1 ; 2 ; 0$ & 2 & $1.72(0.80-3.68)$ \\
\hline
\end{tabular}

n.a.: not applicable due to the low number of cases (see methods).

\# where at least one cardiovascular or AZCERT drug is reported among suspect. interacting or concomitant drugs.

AZCERT List I: Drugs with risk of TdP; List II: Drugs with possible risk of TdP; n.r.: not reported.

* statistically significant ROR (i.e.. 95\% Cl>1). MD: Medical Doctor 


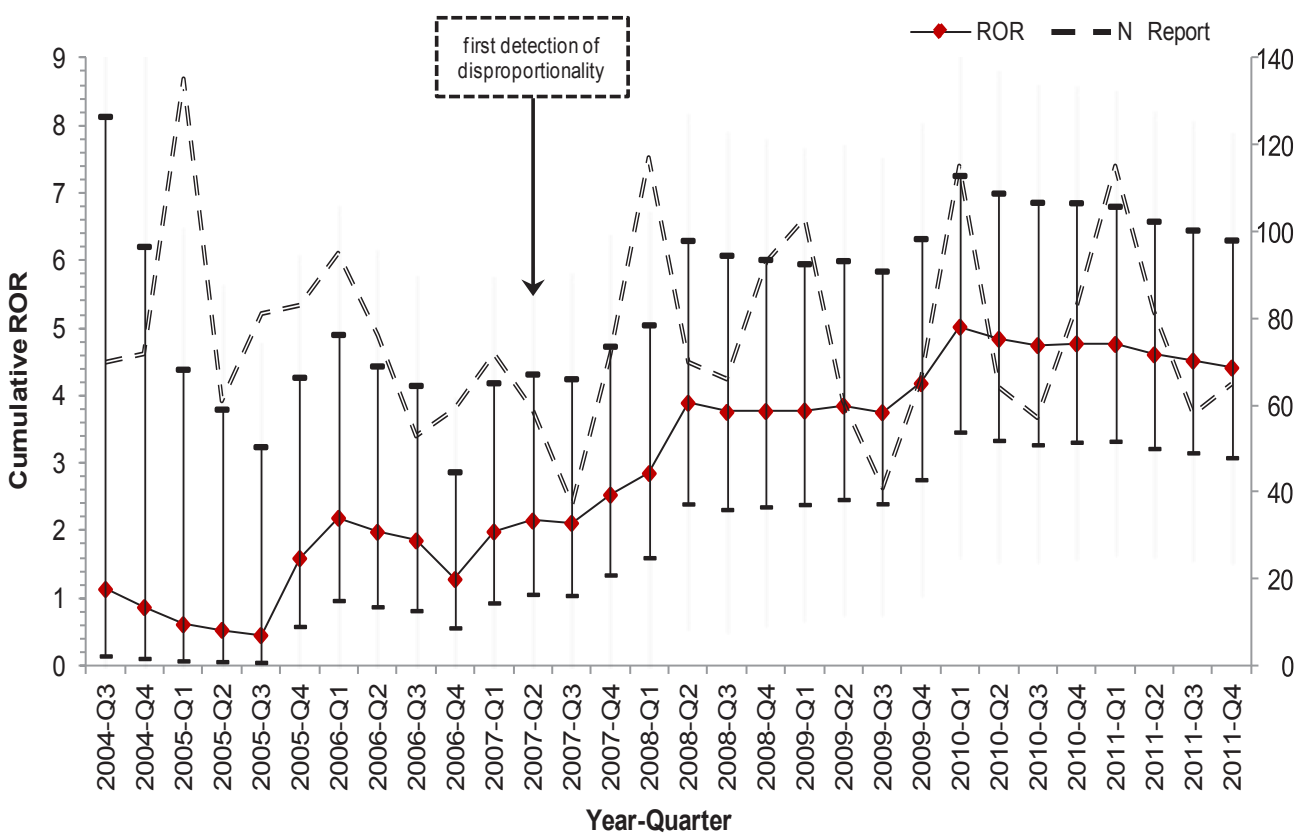

Figure 1: Time trends of cumulative ROR with corresponding $95 \% \mathrm{Cl}$ for TdP and QT abnormalities associated with azithromycin (full line), and relevant total number of spontaneous reports (dotted line). Arrow with box indicates the first appearance of a statistically significant disproportionality (2007-Q2).

associated with disproportionality for TdP-related events, suggesting potential signals of torsadogenicity. This is in line with literature data and information from the Arizona CERT, which considers azithromycin, clarithromycin and erythromycin as having a risk of TdP $[17,10]$.

Based on our data, we believe that including azithromycin in AZCERT list 1 appears prudent, especially in the light of recent emphasis on its novel unlicensed clinical roles (e.g., chronic obstructive pulmonary disease, cystic and non-cystic fibrosis) [18-20]. Our analysis found a remarkable fraction of cases resulting in a fatal outcome, especially for azithromycin, in line with the study by Ray et al. [3]. These finding should be carefully considered, also in the light of a recent observational study showing that azithromycin is the most common prescribed drug (after amiodarone) within 48 hours prior to admission to cardiac unit for QT prolongation and mainly continued during hospitalization [21].

Considering azithromycin a safer option among macrolides in healthy patients appears unjustified, as suggested by the large number of cases occurring in middle-aged patients, who theoretically carry a reduced host-related risk. In addition, the vast proportion of cases with concomitant QT-prolonging drugs (i.e., listed by the Arizona CERT lists 1 and 2) underline the high potential of drug interaction when macrolides are co-administered with other agents undertaking a hepatic metabolism. As a matter of fact, dual intertwined mechanisms (pharmaco-kinetic and-dynamic) are responsible for macrolideassociated TdP: metabolic liability (i.e., the inhibitory effect on CYP3A4) and intrinsic hERG-blocking property [1,2]. Actually, azithromycin showed lower interaction with CYP3A4 as compared to other macrolides whether or not this theoretical advantage represents an effective clinical benefit requires clinical confirmation [22]. In patients with un-modifiable risk factors for TdP occurrence, prescribers should consider alternatives to macrolides.
The clinical implications of our results should be viewed with caution, especially in the light of well-known bias affecting pharmacovigilance data (e.g., quality and completeness of reports, external factors influencing the pattern of reporting, under-reporting and lack of exposure data). Therefore, incidence cannot be determined $[23,24]$. In addition, concomitant reporting of other drugs with known QT liability is only an indicator of potential drug interaction. Other types of information are needed to assess whether or not the interaction actually occurs (e.g., temporal plausibility, which only rarely is recorded in FAERS). Moreover, the supposed safer cardiac profile of azithromycin could have caused the channeling bias of preferring azithromycin in patients at risk of TdP.

Nevertheless, this pharmacovigilance analysis depicts the current situation, which is not influenced by the introduction of novel macrolides and/or antibiotics, recent regulatory measures and, therefore, probably reflects the actual scenario. Our findings corroborate the notion that concomitant drugs with QT liability may significantly impact the occurrence of arrhythmia. Thus, it is imperative that clinicians not only submit adverse event reports to the FDA, but provide complete and accurate information to adequately inform the causality assessment. As previously shown, most patients experiencing TdP had more than two risk factors before the initiation of antibiotic therapy [25]. Therefore, our results strengthen the importance of accurately evaluating medical history (especially concomitant drug administration) to identify patients susceptible to arrhythmia.

In conclusion, our study suggests that in clinical practice azithromycin carries a level of risk similar to other macrolides: the notable proportion of fatal cases and the occurrence of TdP-related events in middle-aged patients strengthen the view that caution is needed before considering azithromycin as a safer therapeutic option among macrolides. Appropriate prescription of all macrolides is therefore vital and should be based on the underlying disease, patient's risk factors, concomitant drugs and local pattern of drug resistance. 
Citation: Raschi E, Poluzzi E, Koci A, Moretti U, Sturkenboom M, et al. (2013) Macrolides and Torsadogenic Risk: Emerging Issues from the FDA Pharmacovigilance Database. J Pharmacovigilance 1: 104. doi:10.4172/2329-6887.1000104

\section{Acknowledgements}

The research leading to these results has received funding from the European Community's Seventh Framework Program (FP7/2007-2013) under grant agreement $n^{\circ} 241679$ - the ARITMO project.

\section{References}

1. Owens RC Jr (2004) QT prolongation with antimicrobial agents: understanding the significance. Drugs 64: 1091-1124.

2. Owens RC Jr, Nolin TD (2006) Antimicrobial-associated QT interval prolongation: pointes of interest. Clin Infect Dis 43: 1603-1611.

3. Ray WA, Murray KT, Hall K, Arbogast PG, Stein CM (2012) Azithromycin and the risk of cardiovascular death. N Engl J Med 366: 1881-1890.

4. De Ponti F, Poluzzi E, Vaccheri A, Bergman U, Bjerrum L, et al. (2002) Nonantiarrhythmic drugs prolonging the QT interval: considerable use in seven countries. Br J Clin Pharmacol 54: 171-177.

5. Raschi E, Poluzzi E, Zuliani C, Muller A, Goossens H, et al. (2009) Exposure to antibacterial agents with QT liability in 14 European countries: trends over an 8-year period. Br J Clin Pharmacol 67: 88-98.

6. Goossens H, Ferech M, Coenen S, Stephens P (2007) Comparison of outpatient systemic antibacterial use in 2004 in the United States and 27 European countries. Clin Infect Dis 44: 1091-1095.

7. Coenen S, Ferech M, Malhotra-Kumar S, Hendrickx E, Suetens C, et al. (2006) European Surveillance of Antimicrobial Consumption (ESAC): outpatient macrolide, lincosamide and streptogramin (MLS) use in Europe.. J Antimicrob Chemother 58: 418-422.

8. Adriaenssens N, Coenen S, Versporten A, Muller A, Minalu G, et al. (2011) European Surveillance of Antimicrobial Consumption (ESAC): outpatient macrolide, lincosamide and streptogramin (MLS) use in Europe (1997-2009). J Antimicrob Chemother 66 Suppl 6: vi37-vi45.

9. Poluzzi E, Raschi E, Moretti U, De Ponti F (2009) Drug-induced torsades de pointes: data mining of the public version of the FDA Adverse Event Reporting System (AERS). Pharmacoepidemiol Drug Saf 18: 512-518.

10. Poluzzi E, Raschi E, Motola D, Moretti U, De Ponti F (2010) Antimicrobials and the risk of torsades de pointes: the contribution from data mining of the US FDA Adverse Event Reporting System. Drug Saf 33: 303-314.

11. Hochberg AM, Pearson RK, O'Hara DJ, Reisinger SJ (2009) Drug-versus-Drug Adverse Event Rate Comparisons: A Pilot Study Based on Data from the US FDA Adverse Event Reporting System. Drug Saf 32: 137-146.

12. Hoffman KB, Kraus C, Dimbil M, Golomb BA (2012) A Survey of the FDA's
AERS Database Regarding Muscle and Tendon Adverse Events Linked to the Statin Drug Class. PLoS One 7: e42866.

13. Poluzzi E, Raschi E, Piccinni C, De Ponti F (2012) Data Mining Applications in Engineering and Medicine. InTech, Croatia.

14. The FDA Adverse Event Reporting System (FAERS): Latest Quarterly Data Files. FDA, USA

15. Roden DM (1998) Taking the "idio" out of "idiosyncratic": predicting torsades de pointes. Pacing Clin Electrophysiol 21: 1029-1034.

16. Raschi E, Piccinni C, Poluzzi E, Marchesini G, De Ponti F (2011) The association of pancreatitis with antidiabetic drug use: gaining insight through the FDA pharmacovigilance database. Acta Diabetol.

17. Shaffer D, Singer S, Korvick J, Honig P (2002) Concomitant risk factors in reports of torsades de pointes associated with macrolide use: review of the United States Food and Drug Administration Adverse Event Reporting System. Clin Infect Dis 35: 197-200

18. Albert RK, Connett J, Bailey WC, Casaburi R, Cooper JA Jr, et al. (2011) Azithromycin for prevention of exacerbations of COPD. N Engl J Med 365: 689-698.

19. Southern KW, Barker PM, Solis-Moya A, Patel L (2012) Macrolide antibiotics for cystic fibrosis. Cochrane Database Syst Rev 11: CD002203.

20. Wong C, Jayaram L, Karalus N, Eaton T, Tong C, et al. (2012) Azithromycin for prevention of exacerbations in non-cystic fibrosis bronchiectasis (EMBRACE) a randomised, double-blind, placebo-controlled trial. Lancet 380: 660-667.

21. Tisdale JE, Wroblewski HA, Overholser BR, Kingery JR, Trujillo TN, et al. (2012) Prevalence of QT interval prolongation in patients admitted to cardiac care units and frequency of subsequent administration of QT interval-prolonging drugs: a prospective, observational study in a large urban academic medical center in the US. Drug Saf 35: 459-470.

22. Abu-Gharbieh E, Vasina V, Poluzzi E, De Ponti F (2004) Antibacteria macrolides: a drug class with a complex pharmacological profile. Pharmacol Res 50: 211-222.

23. Stephenson WP, Hauben M (2007) Data mining for signals in spontaneous reporting databases: proceed with caution. Pharmacoepidemiol Drug Saf 16 : 359-365.

24. Bate A, Evans SJ (2009) Quantitative signal detection using spontaneous ADR reporting. Pharmacoepidemiol Drug Saf 18: 427-436.

25. Justo D, Zeltser D (2006) Torsades de pointes induced by antibiotics. Eur J Intern Med 17: 254-259. 